
17 Orlando, FL, USA.

\title{
DNA damage and macrophage infiltration in the ovaries of the long-lived GH deficient Ames Dwarf and the short-lived bGH transgenic mice
}

\author{
Tatiana Saccon ${ }^{1}$; Monique Tomazele Rovani ${ }^{2}$; Driele Neske Garcia ${ }^{1}$; Jorgea Pradiee ${ }^{3}$; \\ Rafael Gianella Mondadori ${ }^{4}$; Luis Augusto Xavier Cruz ${ }^{4}$, Carlos Castilho Barros ${ }^{5}$; Yimin Fang ${ }^{6}$; \\ Samuel McFadden ${ }^{6}$; Andrzej Bartke ${ }^{6}$; Michal M Masternak ${ }^{7}$; Augusto Schneider ${ }^{5 *}$ \\ ${ }^{1}$ Centro de Desenvolvimento Tecnológico, Biotecnologia, Universidade Federal de Pelotas - RS, \\ Brazil. \\ ${ }^{2}$ Faculdade de Veterinária, Universidade Federal do Rio Grande do Sul, RS, Brazil. \\ ${ }^{3}$ Faculdade de Veterinária, Universidade Federal de Pelotas, RS, Brazil. \\ ${ }^{4}$ Instituto de Biologia, Universidade Federal de Pelotas, Pelotas, RS, Brazil. \\ ${ }^{5}$ Faculdade de Nutrição, Universidade Federal de Pelotas, Pelotas, RS, Brazil. \\ ${ }^{6}$ Departments of Internal Medicine, Southern Illinois University School of Medicine, \\ Springfield, IL, USA. \\ ${ }^{7}$ College of Medicine, Burnett School of Biomedical Sciences, University of Central Florida, \\ *Corresponding author. \\ Email: augusto.schneider@ufpel.edu.br \\ Rua Gomes Carneiro, 1 - Sala 239 \\ CEP 96020-220 \\ Pelotas, RS, Brazil.
}




\section{Abstract}

Objective: The aim of the study was to evaluate the role of growth hormone (GH) in DNA damage, macrophage infiltration and the granulosa cells number of primordial and primary follicles.

Methods: For these experiments six groups of female mice were used. Four groups consisted of Ames dwarf (Prop-1 ${ }^{\mathrm{df}}$, df/df, $\mathrm{n}=12$ ) and their normal littermates $(\mathrm{N} / \mathrm{df}, \mathrm{n}=12)$ mice, between sixteen and eighteen month-old, receiving $\mathrm{GH}(\mathrm{n}=6$ for $\mathrm{df} / \mathrm{df}$, and $\mathrm{n}=6$ for $\mathrm{N} / \mathrm{df}$ mice) or saline injections ( $\mathrm{n}=6$ for $\mathrm{df} / \mathrm{df}$, and $\mathrm{n}=6$ for $\mathrm{N} / \mathrm{df}$ mice). The other two groups consisted of ten to twelve-month-old bGH $(n=6)$ and normal mice $(\mathrm{N}, \mathrm{n}=6)$. Immunofluorescence for DNA damage (anti- $\gamma \mathrm{H} 2 \mathrm{AX}$ ) and macrophage counting (anti-CD68) were performed. Granulosa cells of primordial and primary follicles were counted.

Results: Female df/df mice had lower $\gamma \mathrm{H} 2 \mathrm{AX}$ foci intensity in in both oocytes and granulosa cells of primordial and primary follicles $(\mathrm{p}<0.05)$, indicating less DNA double strand breaks (DSBs). In addition, GH treatment increased DSBs in both df/df and N/df mice. Inversely, bGH mice had higher quantity of DSBs in both oocytes and granulosa cells of primordial and primary follicles $(\mathrm{p}<0.05)$. Df/df mice showed ovarian tissue with less macrophage infiltration than N/df mice $(\mathrm{p}<0.05)$ and GH treatment increased macrophage infiltration $(\mathrm{p}<0.05)$. On the other hand, bGH mice had ovarian tissue with more macrophage infiltration compared to normal mice $(\mathrm{p}<0.05)$. Df/df mice had less granulosa cells on primordial and primary follicles than N/df mice $(\mathrm{p}<0.05)$. GH treatment did not affect the granulosa cells number $(\mathrm{p}>0.05)$. However, bGH mice had an increased number of granulosa cells on primordial and primary follicles compared to normal mice $(\mathrm{p}<0.05)$.

47 Conclusion: The current study points to the role of the GH/IGF-I axis in maintenance of oocyte DNA integrity and macrophage ovarian infiltration in mice.

49 Keywords: Growth hormone; Insulin-like growth factor-1; DNA damage; macrophages; granulosa cells. 


\section{Introduction}

Ames Dwarf mice (df/df) have a defective Prophet of Pit1 (Prop1) gene, which impairs anterior pituitary gland development, resulting in deficient growth hormone $(\mathrm{GH})$ secretion [1]. As a result, df/df mice have very low levels of circulating insulin-like growth factor I (IGF-I), are significantly smaller and live around 30 to $65 \%$ longer than normal littermates (N/df) [2]. In contrast, transgenic mice overexpressing bovine $\mathrm{GH}(\mathrm{bGH})$ have elevated plasma levels of $\mathrm{GH}$, resulting in increased circulating IGF-I levels and adult body size [3, 4]. The lifespan of bGH mice is approximately 50\% shorter than for normal littermates [4]. Collectively, this evidence points to an important role of the GH/IGF-I axis in the aging process.

A progressive decline with aging and consequent depletion of the ovarian primordial follicle reserve is the main cause of the onset of menopause in women [5]. Along with the reduction in follicle numbers, the quality of the remaining oocytes enclosed in these follicles also decreases with age $[5,6]$. It is well established that the age-related decline in fertility is paralleled by a decrease in the ovarian reserve and the increase in chromosomally abnormal conceptions [7]. One of the primary cause of the increased risk to miscarriages with age is frequently attributed to the exponential rise of the oocyte chromosome mis-segregations, leading to karyotypic imbalances and aneuploidies in the offspring [8]. Many of these karyotypic abnormalities result in spontaneous abortion in the first trimester and thus contribute to the high frequency of pregnancy loss during this time window [9]. However, advanced maternal age also poses an increased risk of serious complications that manifest in later pregnancy, these include miscarriage, late fetal and perinatal death, stillbirth, preterm and extreme preterm birth, low birth weight, placenta praevia and pre-eclampsia $[10,11]$.

The GH/IGF-I axis also has a central role in ovarian function [12-14]. Previous studies of our group have shown that middle-age and old GH-deficient df/df mice have a larger ovarian primordial follicle reserve than N/df mice $[15,16]$. This indicates that increased longevity is correlated with increased reproductive lifespan. Furthermore, bGH mice had a smaller ovarian reserve than normal mice [17], showing that the GH/IGF-I axis has a central role in the reproductive lifespan. In spite of the evidences of the role of GH/IGF-I in the preservation of the ovarian reserve little is known about its effects on the other ovarian parameters as the mice ages. 
Several factors can influence fertility during aging. The adverse effects of DNA damage caused by environmental factors on somatic cells have been extensively reported [18]. Mammalian oocytes enclosed in primordial follicles remain arrested in prophase I of meiosis up to several decades in some species, including humans [19, 20]. This long period of dormancy which oocyte are submit through increases the chances of accumulating DNA damage, as shown in female mice and human, both of which, accumulate double strand breaks (DSBs) in primordial follicle oocytes with aging [21]. Substantial damage occurring throughout meiosis can have serious consequences if an adequate cellular response is not activated, and may result in infertility or development of defective embryos that are unable to result in full-term pregnancy $[22,23]$. As a reaction to DSBs, kinases are known to phosphorylate histone $2 \mathrm{Ax}(\gamma \mathrm{H} 2 \mathrm{AX})$ on serine 139 [24, 25]. This post-transcriptional modification at the lesion site provides a platform for the ataxia-telangiectasia mutated (ATM)-mediated DNA damage signaling pathway to regulates the repair of DNA DSBs [26].

In addition to DNA damage, inflammation also increases with age and can negatively affect fertility [27]. Interleukin 1 deficient female mice have more primordial follicles and increased fertility than control females [28]. One of our previous studies identified by RNASeq that old $\mathrm{df} / \mathrm{df}$ mice have more than 150 down-regulated gene ontology terms related to the inflammatory/immune response compared to N/df mice [29], pointing to a role of inflammation in ovarian aging. It is well known that adipose tissue and blood levels of pro-inflammatory cytokines, interleukin 6 (IL-6) and tumor necrosis factor alpha (TNF- $\alpha$ ) are reduced in df/df mice $[30,31]$. This reduced inflammation is believed to be one of the main reasons for the increased longevity of df/df mice [32]. Given the background, the presence of inflammatory cells within the ovaries is an important parameter, as macrophages are the most numerous immune cells within the ovary [33]. Inflammation can result in oxidative stress, reducing cellular antioxidant capacity, leading to overproduction of free radicals that react with cell membrane fatty acids and proteins impairing their function permanently [34]. Furthermore, excess free radicals can increase DNA damage, a predisposing factor for several age-related disorders [35].

In mammalian ovaries, the majority of oocytes are meiotically arrested and surrounded by a layer of flattened somatic granulosa cells, a structure known as primordial follicle [36, 37]. For the purpose to ensure the proper reproductive lifespan, most of the oocytes are maintained in this quiescent state within primordial follicles [38]. Granulosa cells play a fundamental role in 
112 initiating the growth of primordial follicles [38]. The number of granulosa cells can be a useful 113 marker of follicular activation and development [39]. Several molecular networks mediate the 114 interaction between somatic cells and germ cells in controlling the development of dormant 115 mammalian oocytes [38, 40]. For example, granulosa produced mammalian target of rapamycin 116 (mTOR) stimulates Kit which in the oocyte stimulates the phosphoinositide 3-kinase 117 (Pi3k)/protein kinase B (Akt1) pathway and the transcription factor Forkhead Box O3a (FoxO3a) 118 phosphorylation resulting in primordial follicle activation [38, 41, 42]. We have previously 119 shown that $\mathrm{GH}$ has a central role in primordial follicle activation through activation of FoxO3a 120 [16], however, to date little is known about its effects on granulosa cells number in primordial 121 and primary follicles.

122 Based on this evidence, the aim of the study was to evaluate the DNA damage, macrophage infiltration and the granulosa cells number of primordial and primary follicles in the ovaries of aged df/df and N/df mice treated with exogenous GH and bGH transgenic mice.

Materials and methods:

\section{Animals and treatment}

For these experiments six groups of female mice were used. Four groups consisted of Ames dwarf (Prop-1 ${ }^{\mathrm{df}}$, df/df, $n=12$ ) and their normal littermates (N/df, $\left.n=12\right)$ mice, between sixteen and eighteen month-old, receiving $\mathrm{GH}(\mathrm{n}=6$ for $\mathrm{df} / \mathrm{df}$, and $\mathrm{n}=6$ for $\mathrm{N} / \mathrm{df}$ mice) or saline injections ( $\mathrm{n}=6$ for $\mathrm{df} / \mathrm{df}$, and $\mathrm{n}=6$ for $\mathrm{N} / \mathrm{df}$ mice). The other two groups consisted of ten to twelve-month-old bGH $(n=6)$ and normal mice $(\mathrm{N}, \mathrm{n}=6)$. Mice were maintained under temperature $\left(22 \pm 2{ }^{\circ} \mathrm{C}\right)$ and humidity (40-60\%) controlled conditions. All experiments were approved by the Ethics Committee for Animal Experimentation from the University of Southern Illinois, IL, USA. Mice treated with GH received recombinant porcine GH subcutaneous injections ( $4 \mu \mathrm{g} / \mathrm{g}$ of body weight; Alpharma, Inc., Victoria, Australia) twice daily, beginning at fourteen months of age for six weeks. Control mice received saline injections in the same way as $\mathrm{GH}$ treated mice. After six weeks of treatment, mice were kept two more weeks in the same conditions until euthanasia. The GH treatment used in the current study was proven effective for increasing serum IGF-I concentrations and body weight gain in previous studies using the same dose and treatment length in young (one month-old) [43, 44], middle age (five month-old) [45] 
and old mice (sixteen month-old)[43]. Body weights were measured before the first GH injection and at the end of the six-week treatment to confirm effectiveness of the treatment.

\section{Tissue collection and processing}

The mice were anesthetized and euthanized after fasting for $12 \mathrm{~h}$ and the pair of ovaries was collected, dissected from surrounding adipose tissue and placed in $10 \%$ formalin buffered solution. After that, the ovaries were removed from the formalin solution, dehydrated in alcohol, cleared in xylene and embedded individually in Paraplast Plus (Sigma Chemical Company®, St. Louis, MO, USA). One ovary of the pair was then serially sectioned at $5 \mu \mathrm{m}$ using a semiautomated rotary microtome (RM2245, Leica Biosystems, San Diego, CA, USA). Sections were randomly selected for immunohistochemistry analysis using slides impregnated with 3\% organosilane (Sigma Chemical Company®, St. Louis, MO, USA) in ethanol.

\section{Immunofluorescence}

For immunofluorescence analysis, the ovarian samples were deparaffinized with xylene and rehydrated with graded alcohols. The primary monoclonal antibodies were obtained from Abcam (Abcam Plc, Cambrigde, UK) and diluted in 1.5\% BSA solution. The anti-gamma H2AX $(\gamma \mathrm{H} 2 \mathrm{AX})$ phospo S139 antibody (ab11174), to indicate DNA damage [21] and anti-CD68 antibody (ab955), to indicate the presence of macrophage [46] were used at a final dilution of 1:500. The blockage of the endogenous peroxidase activity was achieved with hydrogen peroxide blocking solution, while the antigen recovery was performed in humid heat, during 3 min after the boiling point, in citrate solution at $\mathrm{pH}$ 6.0. Non-specific background staining was reduced by covering the tissue sections that received protein block with BSA and goat serum. Thereafter, slides were incubated overnight with the primary antibody in a humid chamber at $4^{\circ} \mathrm{C}$. The slides with anti- $\gamma \mathrm{H} 2 \mathrm{AX}$ and anti-CD68 antibodies were incubated for 1 hour with secondary Alexa Fluor® 488 (ab150113) and Hoechst (ab228550) for nuclei stanning during 15 min. The slides were mounted with a drop of mounting medium under coverslips. The images of the follicles were captured by confocal microscope (Olympus FluoView ${ }^{\mathrm{TM}}$ 1000). Fluorescence intensity quantification for $\gamma \mathrm{H} 2 \mathrm{AX}$ and macrophage counting was performed by image analysis software Image $\mathrm{J} \circledast$ [47]. $\gamma \mathrm{H} 2 \mathrm{AX}$ and granulosa cells number was measured in 3 
174 per sections of ovary. Each mouse had a total of 4 random sections from the center of the ovary 175 counted. The number of granulosa cells was counted as visible nuclei using the Hoechst 176 (ab228550) for nuclei staining.

177

178

179

180

181

182

183

184

185

186

187

188

189

190

191

192

193

194

195

196

197

198

199

200

201

202

203

204

\section{Statistical analyzes}

All statistical analyzes were performed using Graphpad Prism 6 (Graphpad Inc., La Jolla, CA, USA). Two-way ANOVA was used for comparing the fluorescence intensity, number of granulosa cells and macrophage number of $\mathrm{df} / \mathrm{df}$ and N/df mice (effect of the genotype, treatment with GH and the interaction). T-test was performed for comparing fluorescence intensity, number of granulosa cells and macrophage number between bGH and normal mice. A P value lower than 0.05 was considered as statistically significant.

\section{Results}

Female df/df mice had less DNA DSBs in primordial and primary follicles, as indicated by by lower fluorescence for $\gamma \mathrm{H} 2 \mathrm{AX}$ in oocytes from primordial $(p<0.0001)$ and primary $(p$ $<0.0001$ ) follicles compared to $\mathrm{N} / \mathrm{df}$ mice. Also, df/df mice had reduced $\gamma \mathrm{H} 2 \mathrm{AX}$ intensity in granulosa cells of primordial $(p=0.0299)$ and primary $(p=0.0002)$ follicles. GH treatment increased DSBs in both df/df and N/df mice, oocytes from primordial $(p<0.0001)$ and primary $(p$ $<0.0001)$ follicles. Also, GH treatment increased DSBs in granulosa cells from primordial $(p$ $<0.0001)$ and primary $(p<0.0001)$ follicles in both $\mathrm{df} / \mathrm{df}$ and N/df mice. In the other hand, bGH mice had higher quantity of DSBs (Figure 1). The fluorescence intensity for $\gamma \mathrm{H} 2 \mathrm{AX}$ in oocytes from primordial $(p=0.0001)$ and primary $(p<0.0001)$ follicles were higher in bGH mice compared to normal mice. Also, granulosa cells from primordial $(p<0.0001)$ and primary $(p=$ 0.0466) follicles had a higher fluorescence intensity for $\gamma \mathrm{H} 2 \mathrm{AX}$ in bGH mice (Figure 2). Representative images of anti- $\gamma \mathrm{H} 2 \mathrm{AX}$ intensity in oocyte and granulosa cells of primordial and primary follicles are shown in Figure 3.

Df/df mice had ovarian tissue with the reduced macrophage infiltration $(p<0.0001)$ and the GH treatment on $\mathrm{df} / \mathrm{df}$ and N/df mice increased macrophage infiltration $(p=0.0007)$. On the other hand, bGH mice had ovarian tissue with increased macrophage infiltration $(p<0.0001)$ (Figure 4). 
Df/df mice had less granulosa cells on primordial $(p=0.0002)$ and primary $(p<0.0001)$ follicles than N/df mice. GH treatment did not change the number of granulosa cells of primordial and primary follicles $(p>0.05)$. However, bGH mice had an increased number of granulosa cells on primordial $(p<0.0001)$ and primary $(p<0.0001)$ follicles compared to normal mice (Table 1).

\section{Discussion}

The current study points to the role of the GH/IGF-I axis in maintenance of oocyte DNA integrity and ovarian macrophage infiltration. GH-deficient $\mathrm{df} / \mathrm{df}$ mice are known for being smaller-sized and living longer and healthier life than normal littermate controls [2, 48], in the other hand, bGH mice are known for a bigger-sized body and short lifespan [4]. Our previous work [16], had shown that df/df mice have more ovarian primordial follicles than normal littermates, and that $\mathrm{GH}$ treatment increased primordial follicle activation and reduced the ovarian reserve. Additionally, we shown that bGH mice have decreased primordial follicle reserve. Therefore, our previous work shown increased ovarian reserve depletion promoted by $\mathrm{GH}$, and our current findings also point to a role for GH in increasing oocyte and granulosa DNA DSBs and inflammation. This can suggest that beyond preserving the reserve for longer periods, GH/IGF-I deficiency also accelerates oocyte DNA damage which can impact fertility.

Oocyte and granulosa cells from primordial and primary follicles DNA DSBs were reduced in old GH-deficient df/df mice, while GH exogenous treatment increased accumulation of DNA DSBs. On the contrary, bGH mice had increased accumulation of DNA DSBs in oocytes and granulosa cells from primordial and primary follicles. DNA damage is a challenge that all somatic and germline cells are exposed during their lifetime [49]. Cells respond to DSBs as a serious threat to their integrity, activating DNA damage checkpoints (DDC) and reacting with a DNA damage response resulting in cell cycle arrest as a downstream effect, allowing activation of repair mechanisms [50]. DSBs trigger a DDC response by activating two major kinases, i.e. ATM and ataxia telangiectasia and Rad3-related (ATR) [51, 52]. Taking advantage of the cell cycle arrest, the cell can then repair the damaged DNA. At the DNA damage site, ATM gives rise to the phosphorylated form of the H2AX histone, which acts as a catalyst for the recruitment of the necessary checkpoint and repair factors [53]. Snell dwarf mice, 
endocrinologically identical to $\mathrm{df} / \mathrm{df}$ mice, have increased cellular DNA repair capacity and upregulation of several DNA repair-related genes compared to normal littermates [54]. Our data, therefore, points to a role for the GH/IGF-I axis in DNA damage in mice oocytes and surrounding granulosa cells from the ovarian reserve. Mice and human oocytes accumulate DNA DSBs with age which contributes to reproductive aging [21]. Additionally, granulosa cells are essential for oocyte growth and activation, and others have also shown increased DNA DSBs in monkey granulosa cells with age [55]. Therefore, here for the first time we suggest that GH/IGFI, which has a central role in somatic and gonadal aging, can also prevent accumulation of DNA damage, further contributing to the younger phenotype observed in GH-deficient mice. The decrease of DNA DSBs repair is also associated with accumulation of DSBs in human and mouse oocytes [21]. The expression of Breast Cancer 1 protein (BRCA1) and other key genes in the ATM-pathway decline with age in human oocytes, increasing DNA DSBs [21]. We previously observed a three-fold reduction in BRCAl gene expression with aging in $\mathrm{N}$ mice, although its expression did not change with aging in df/df mice [29], further contributing to DSB accumulation coordinated by GH/IGF-I axis. Overall, this work shows that GH/IGF-I deficiency beyond preserving the ovarian reserve for longer periods, can also contribute to maintaining oocyte DNA integrity, increasing the chances of successful pregnancy in older ages.

Old df/df mice exhibited reduced ovarian macrophage infiltration, and the treatment with $\mathrm{GH}$ in adult life increased the number of macrophages in the ovary. In the other hand, bGH mice had increased macrophage infiltration. These evidences suggest the GH/IGF-I axis as central to regulating ovarian macrophage infiltration. The long-living $\mathrm{df} / \mathrm{df}$, growth hormone receptor knockout mice (GHRKO) and calorie restricted mice have all been extensively characterized as having a reduced pro-inflammatory profile, which may represent one of the major mechanisms promoting increased insulin sensitivity and extended longevity in these mice [32]. Macrophages located in the ovaries, by secreting growth factors and/or cytokines, may play a synergistic role in stimulating cellular proliferation and follicle growth [56]. Some of the macrophage $\square$ derived factors that are known to impact follicular growth are hepatocyte growth factor (HGF), basic fibroblast growth factor (bFGF), tumor necrosis factor (TNF) $\alpha$ and $\beta$, and IGF-I [57-59]. On the other hand, prolonged exposure (both short-term and long-term) to a high-fat diet in young adult female mice reduced primordial follicle numbers, compromised fertility, produced higher systemic proinflammatory cytokine levels, and increased ovarian macrophage infiltration in the 
stroma, independent of obesity [46]. Pro-inflammatory Interleukin 1 deficient female mice have more primordial follicles and increased fertility than control females [28]. Also, LPS exposure in mice reduced the primordial follicle pool mediated by TLR4 [60], indicating the role of inflammation on ovarian reserve depletion. Our ovarian transcriptome study showed that the top 150 down-regulated terms between old $\mathrm{df} / \mathrm{df}$ and old $\mathrm{N}$ mice were related to the inflammatory/immune response [29]. It was observed that macrophage chemotaxis, macrophage activation and macrophage differentiation were also among the top down-regulated biological processes in old $\mathrm{df} / \mathrm{df}$ compared to old $\mathrm{N}$ mice. For that, these findings point to an association between the GH/IGF-1 axis and the inflammation on ovaries of old df/df mice. bGH mice have no data in ovarian inflammation, however, these transgenic mice have an increase proinflammatory profile in kidney [61] and age related increased pro-inflammatory markers in blood [62]. Another study with osteopetrotic mice showed reduced numbers of mature macrophages due to a natural mutation in the CSF $\square 1$ gene [63]. These osteopetrotic mice have reduced numbers of ovarian macrophages, which is not still clear if this is cause or effect of the decreased follicle growth on these mice. Our evidences provide an interesting connection between ovarian longevity and macrophage population coordinated by the GH/IGF-I axis, however, more studies are necessary to understand the role of macrophages in the ovarian aging.

The microenvironment within follicles and ovaries also could be influenced by GH/IGF-I deficiency. Particularly, granulosa cells and cumulus cells are intimately connected with oocytes, and both play critical roles in oocyte growth, follicular development and could also influence oocyte quality $[64,65]$. Oocyte growth is accompanied by the active accumulation of mRNA, proteins, and lipids, and by modifications of chromatin configuration and DNA methylation status, and these molecular reactions require enough energy [66]. The number of granulosa cells in follicles is associated with the energy sufficiency of oocytes [66], which can affect oocyte and follicle growth. A complete set of molecular networks that mediate the interaction between somatic cells and germ cells in controlling the development of dormant mammalian oocytes had been described. The model proposed by Zhang et al. (2014) has two key steps. The first step is the mTOR complex 1 (mTORC1) signaling in granulosa cells that acts as the key decisionmaking process regarding whether or not a primordial follicle will be activated [38]. The second step involves the tightly regulated communication channel from the granulosa cells to the oocytes via KITL-KIT to trigger the awakening of the oocyte through FoxO3a phosphorylation 
298 [38]. It is proposed that these processes ensure the progressive activation of a limited number of 299 primordial follicles throughout the reproductive lifespan. Thus, it is likely that follicular 300 activation is initiated by molecular and cellular changes in the granulosa cells that are followed 301 by awakening of the dormant oocytes. Our study shows that $\mathrm{df} / \mathrm{df}$ mice had fewer granulosa cells 302 surrounding oocytes than N/df mice in both primordial and primary follicles, supporting the late 303 activation of primordial follicle observed for $\mathrm{df} / \mathrm{df}$ mice in a previously study [16]. Also, bGH 304 mice had a larger number of granulosa cells compared to normal mice, suggesting that granulosa 305 cell number is an indication of successful follicle activation and development, since bGH mice presented less primordial follicles than normal mice [16]. This is in line with our previous work 307 showing that FoxO3a activation is regulated by the GH/IGF-I axis in mice primordial and 308 primary oocytes. Therefore, regulation of granulosa cell number by GH/IGF-I can be a critical 309 point in determining ovarian lifespan.

\section{Conclusion}

In conclusion, the present study indicates that GH/IGF-I is associated to oocyte and 314 granulosa cell DNA damage and ovarian macrophage infiltration. Adding to our previous work, 315 the current study demonstrates that beyond preserving the ovarian primordial reserve, GH/IGF-I 316 deficiency prevents DNA damage and inflammation, both parameters linked to fertility in older 317 ages. Overall, these important observations confirm, that delayed aging phenotype in these 318 unique long-living mice, suggesting a beneficial characteristic of longevity in the reproductive organs of female df/df mice coordinated by GH/IGF-I levels. 
A

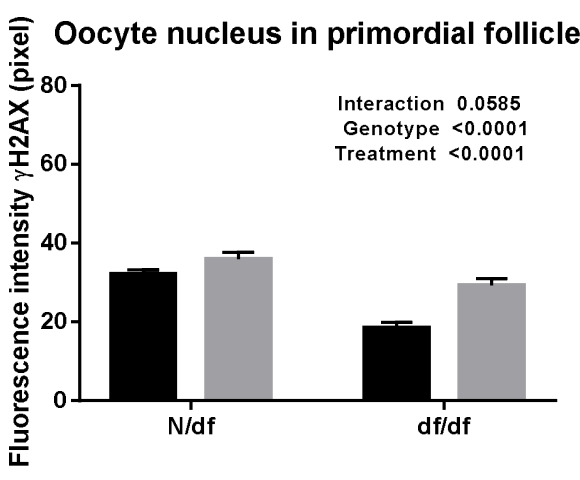

C

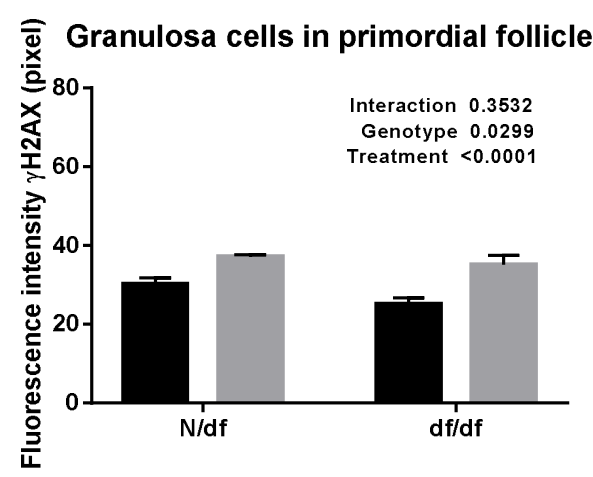

B

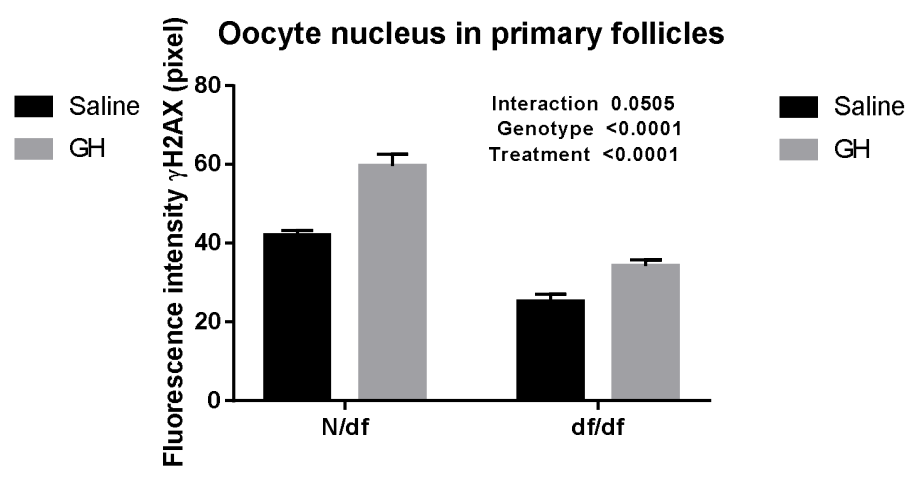

$\mathbf{D}$

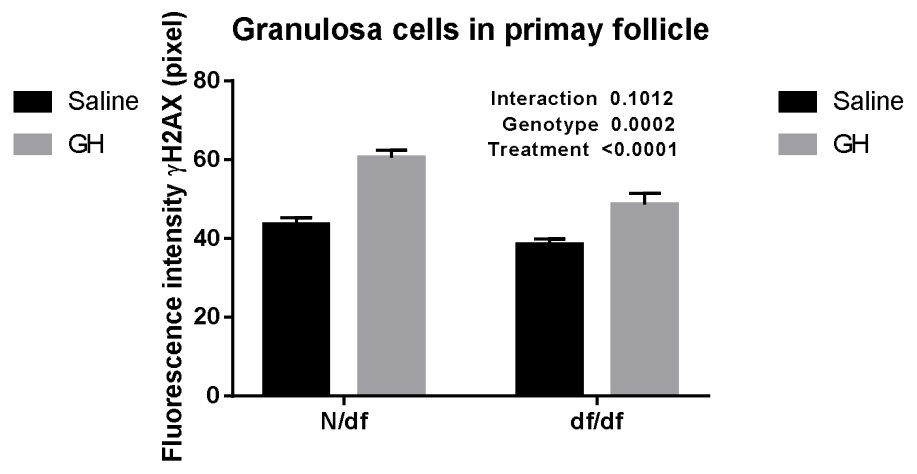

325 Figure 1. Fluorescence intensity of $\gamma \mathrm{H} 2 \mathrm{AX}$ immunostaining in oocytes nucleus of primordial 326 follicle $(\mathrm{n}=18, \mathrm{~A})$, oocyte nucleus of primary follicle $(\mathrm{n}=18, \mathrm{~B})$ and granulosa cell nuclei from 327 primordial $(n=18, C)$ and primary follicles $(n=18, D)$ of $\mathrm{N} / \mathrm{df}$ and $\mathrm{df} / \mathrm{df}$ mice treated with 328 exogenous GH or saline. Data presented as media \pm SEM. 


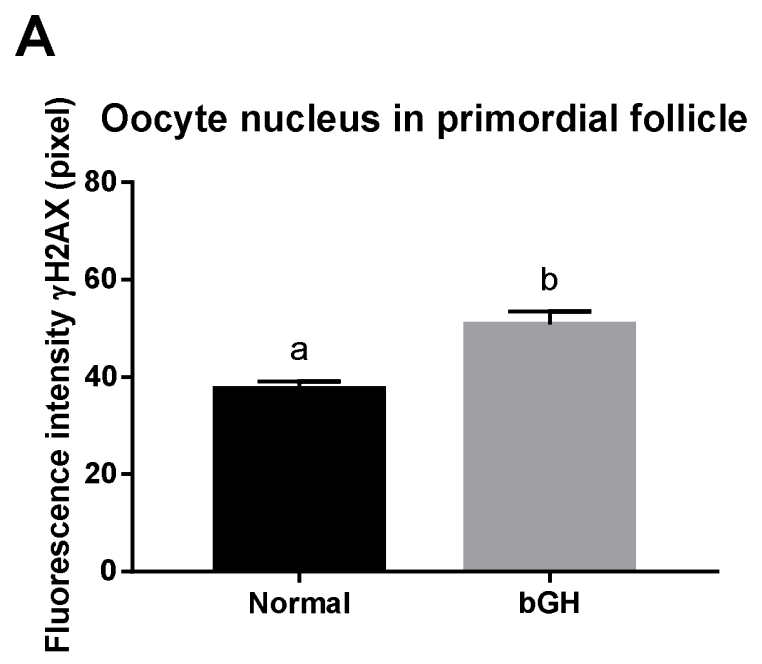

\section{B}

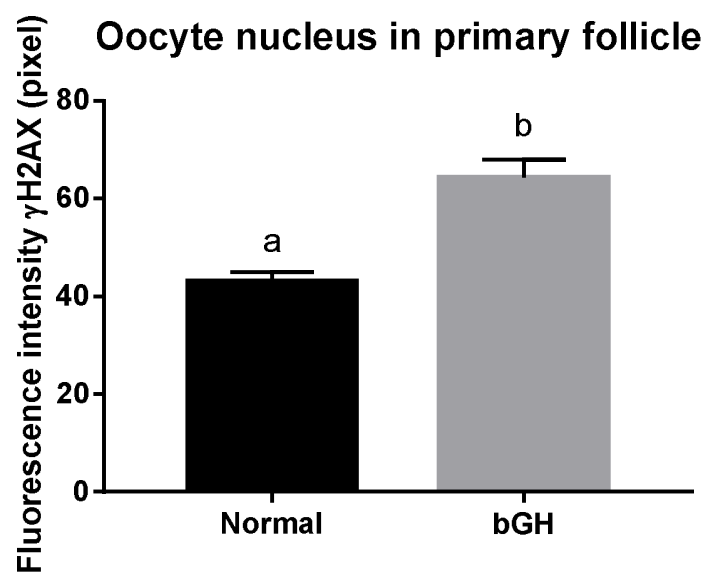

C

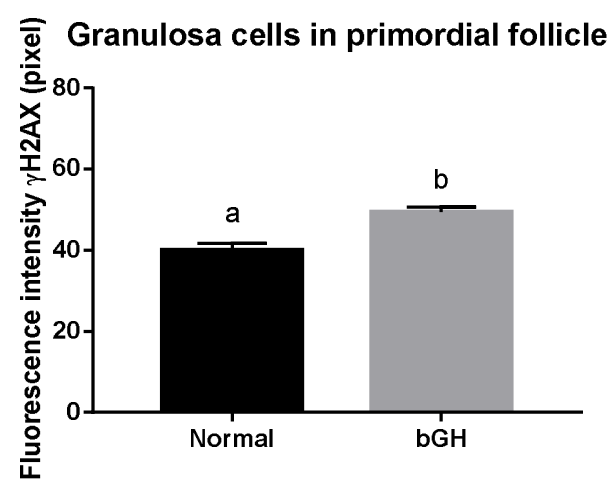

D

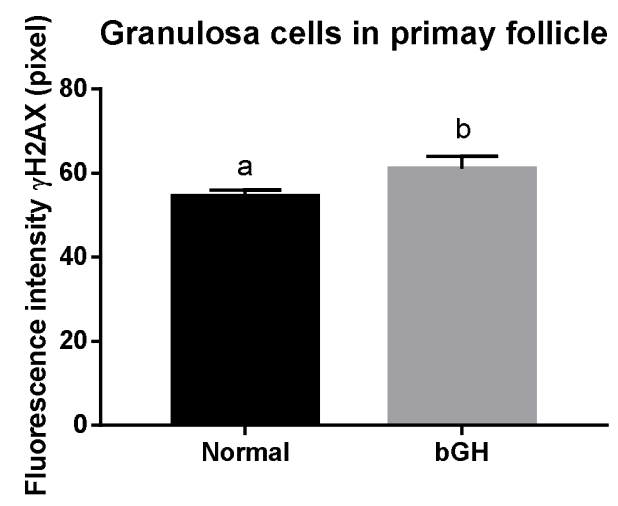

332 Figure 2. Fluorescence intensity of $\gamma \mathrm{H} 2 \mathrm{AX}$ immunostaining in oocytes nucleus of primordial

333 follicle $(\mathrm{n}=18, \mathrm{~A})$, oocyte nucleus of primary follicle $(\mathrm{n}=18, \mathrm{~B})$ and granulosa cell nuclei from

334 primordial $(\mathrm{n}=18, \mathrm{C})$ and primary follicles $(\mathrm{n}=18, \mathrm{D})$ of normal and bGH transgenic mice. Different letters indicate significant differences $(p<0.05)$. Data presented as media \pm SEM. 
A Primordial follicles
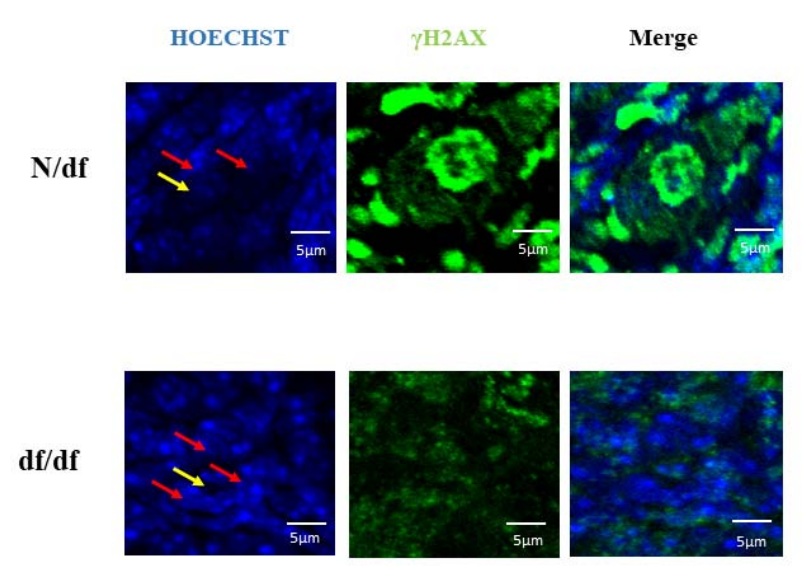

B

Primordial follicles

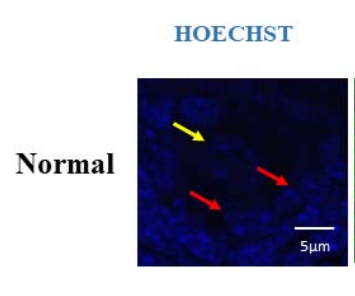

$\gamma \mathrm{H} 2 \mathrm{AX}$
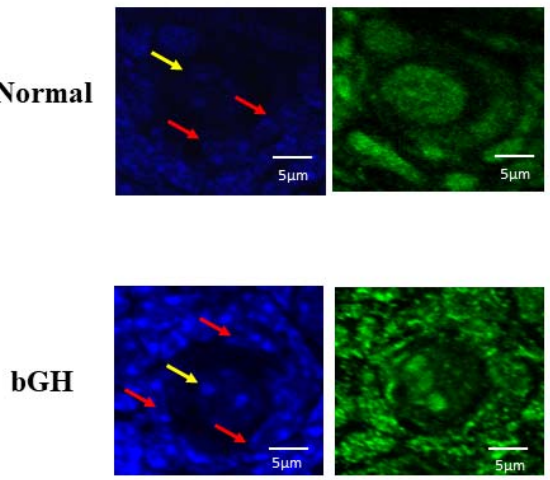

Merge
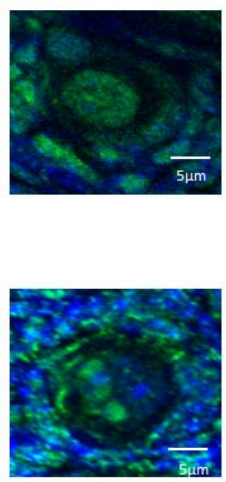

Primary follicles
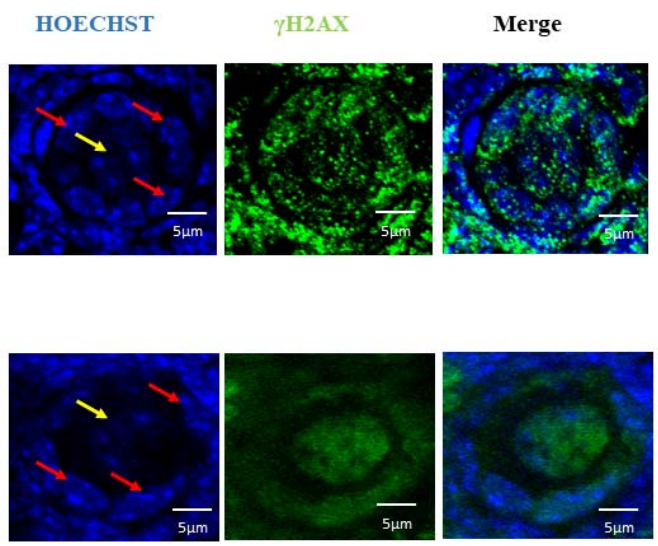

HOECHST
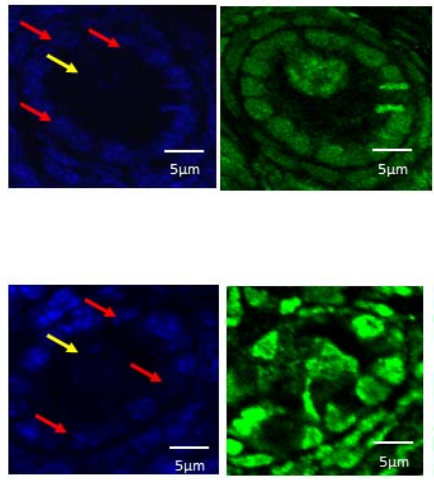

$\gamma \mathrm{H} 2 \mathrm{AX}$

Merge
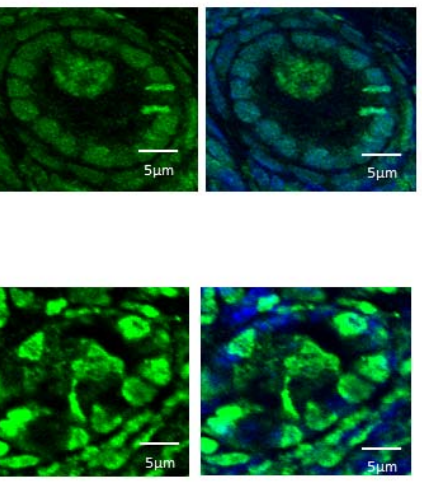

340 Figure 3. Representative images of immunofluorescence of anti- $\gamma \mathrm{H} 2 \mathrm{AX}$ in primordial and 341 primary follicles of $\mathrm{N} / \mathrm{df}$ and $\mathrm{df} / \mathrm{df}$ mice (A); and normal and bGH mice (B). Blue images 342 represent Hoechst (staining genetic material) and green represent anti- $\gamma \mathrm{H} 2 \mathrm{AX}$ staining $\gamma \mathrm{H} 2 \mathrm{AX}$ 
bioRxiv preprint doi: https://doi.org/10.1101/2020.01.19.911677; this version posted January 19, 2020. The copyright holder for this preprint (which was not certified by peer review) is the author/funder. All rights reserved. No reuse allowed without permission.

343 protein. Merged images are showed as both Hoechst and anti- $\gamma \mathrm{H} 2 \mathrm{AX}$ staining combination. Red

344 arrows represent some granulosa cells and yellow arrow represents oocyte nucleus.

345 

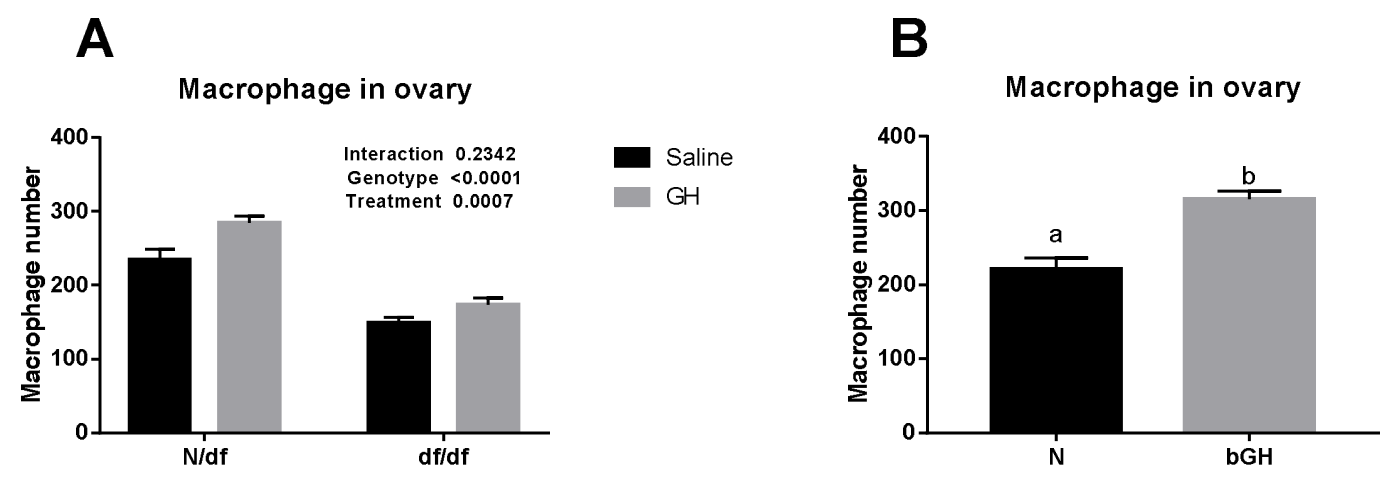

347
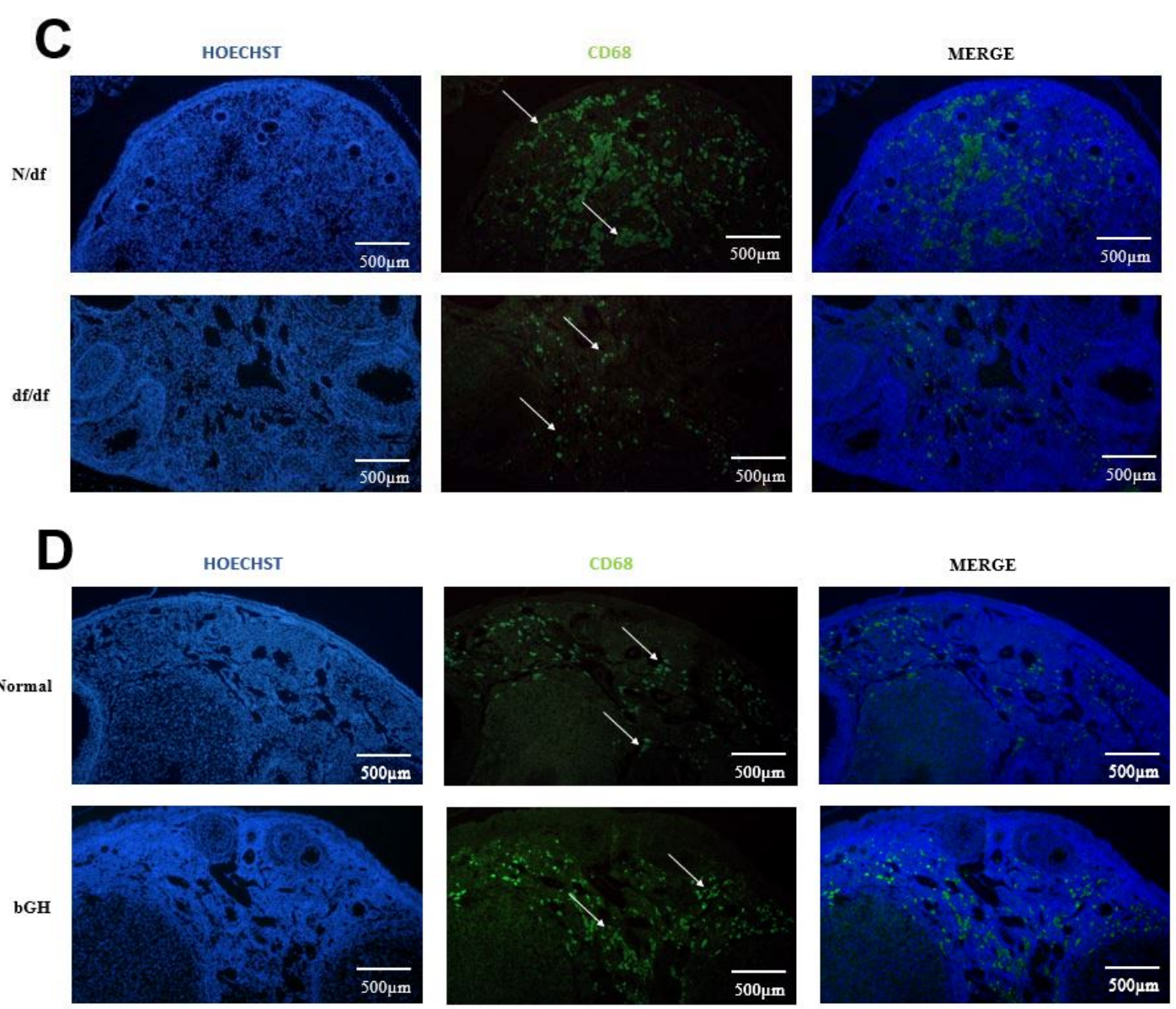

351 Figure 4. Macrophage number per ovarian section in $\mathrm{N} / \mathrm{df}(\mathrm{n}=24)$ and $\mathrm{df} / \mathrm{df}(\mathrm{n}=24)$ mice

352 treated with exogenous GH or saline (A); and macrophage number on ovarian section of normal

$353(\mathrm{n}=24)$ and bGH $(\mathrm{n}=24)$ transgenic mice $(\mathrm{B})$. Representative images of macrophage 
bioRxiv preprint doi: https://doi.org/10.1101/2020.01.19.911677; this version posted January 19, 2020. The copyright holder for this preprint (which was not certified by peer review) is the author/funder. All rights reserved. No reuse allowed without permission.

354 infiltration on ovarian section of N/df and df/df (C); normal and bGH transgenic mice (D). White 355 arrow indicates macrophage staining. Different letters indicate significant differences $(p<0.05)$. 
357 Table 1. Number of granulosa cells of df/df and N/df mice treated with exogenous GH and bGH 358 transgenic mice.

\begin{tabular}{|c|c|c|c|c|c|c|c|}
\hline & & & & & \multicolumn{3}{|c|}{$p$-Value ${ }^{\mathrm{a}}$} \\
\hline \multirow{3}{*}{ Follicle Class } & \multicolumn{4}{|c|}{ Granulosa cell number $(n=18)($ SEM) } & Genot. & Treat. & $\begin{array}{c}\text { Genot* } \\
\text { Treat }\end{array}$ \\
\hline & \multicolumn{2}{|c|}{ N/df } & \multicolumn{2}{|c|}{ df/df } & & & \\
\hline & Saline & GH & Saline & GH & & & \\
\hline Primordial Follicles & $6.0( \pm 0.2)$ & $6.2( \pm 0.2)$ & $4.8( \pm 0.2)$ & $5.4( \pm 0.2)$ & $0.0002 *$ & 0.1389 & 0.5425 \\
\hline \multirow[t]{3}{*}{ Primary Follicles } & $10.3( \pm 0.4)$ & $11.4( \pm 0.6$ & $8.8( \pm 0.3)$ & $8.7( \pm 0.3)$ & $<0.0001 *$ & 0.2796 & 0.1590 \\
\hline & & & & & & & $p$-Value ${ }^{b}$ \\
\hline & \multicolumn{2}{|c|}{ Normal } & \multicolumn{2}{|c|}{ bGH } & & & \\
\hline Primordial Follicles & \multicolumn{2}{|c|}{$5.4( \pm 0.4)$} & \multicolumn{2}{|c|}{$9.9( \pm 1.1)$} & & & $<0.0001 *$ \\
\hline Primary Follicles & \multicolumn{2}{|c|}{$8.2( \pm 0.3)$} & \multicolumn{2}{|c|}{$17.7( \pm 0.9)$} & & & $<0.0001 *$ \\
\hline
\end{tabular}




\section{References}

[1] M.W. Sornson, W. Wu, J.S. Dasen, S.E. Flynn, D.J. Norman, S.M. O'Connell, I. Gukovsky, C. Carriere, A.K. Ryan, A.P. Miller, L. Zuo, A.S. Gleiberman, B. Andersen, W.G. Beamer, M.G. Rosenfeld, Pituitary lineage determination by the Prophet of Pit-1 homeodomain factor defective in Ames dwarfism, Nature 384(6607) (1996) 327-33.10.1038/384327a0.

[2] V. Chandrashekar, A. Bartke, Effects of age and endogenously secreted human growth hormone on the regulation of gonadotropin secretion in female and male transgenic mice expressing the human growth hormone gene, Endocrinology 132(4) (1993) 14828.10.1210/endo.132.4.8462447.

[3] A. Blackburn, R.A. Dressendorfer, W.F. Blum, M. Erhard, G. Brem, C.J. Strasburger, E. Wolf, Interactions of insulin-like growth factor (IGF)-II and growth hormone in vivo: circulating levels of IGF-I and IGF-binding proteins in transgenic mice, Eur J Endocrinol 137(6) (1997) 701-8.10.1530/eje.0.1370701.

[4] E. Wolf, E. Kahnt, J. Ehrlein, W. Hermanns, G. Brem, R. Wanke, Effects of long-term elevated serum levels of growth hormone on life expectancy of mice: lessons from transgenic animal models, Mech Ageing Dev 68(1-3) (1993) 71-87.10.1016/0047-6374(93)90141-d.

[5] M.J. Faddy, R.G. Gosden, A. Gougeon, S.J. Richardson, J.F. Nelson, Accelerated disappearance of ovarian follicles in mid-life: implications for forecasting menopause, Hum Reprod 7(10) (1992) 1342-6.10.1093/oxfordjournals.humrep.a137570.

[6] A.N. Hirshfield, Relationship between the supply of primordial follicles and the onset of follicular growth in rats, Biol Reprod 50(2) (1994) 421-8.10.1095/biolreprod50.2.421.

[7] S.E. McGowan, D.M. McCoy, Platelet-derived growth factor-A regulates lung fibroblast Sphase entry through p27(kip1) and FoxO3a, Respir Res 14 (2013) 68.10.1186/1465-9921-14-68.

[8] L. Woods, V. Perez-Garcia, J. Kieckbusch, X. Wang, F. DeMayo, F. Colucci, M. Hemberger, Decidualisation and placentation defects are a major cause of age-related reproductive decline, Nat Commun 8(1) (2017) 352.10.1038/s41467-017-00308-x.

[9] N. Ashaat, A. Husseiny, Correlation between missed abortion and insertional translocation involving chromosomes 1 and 7, Iran J Reprod Med 10(1) (2012) 15-22.

[10] E.L. Abel, M. Kruger, L. Burd, Effects of maternal and paternal age on Caucasian and Native American preterm births and birth weights, Am J Perinatol 19(1) (2002) 49-54.10.1055/s2002-20173.

[11] B. Jacobsson, L. Ladfors, I. Milsom, Advanced maternal age and adverse perinatal outcome, Obstet Gynecol 104(4) (2004) 727-33.10.1097/01.AOG.0000140682.63746.be.

[12] A. Bachelot, P. Monget, P. Imbert-Bollore, K. Coshigano, J.J. Kopchick, P.A. Kelly, N. Binart, Growth hormone is required for ovarian follicular growth, Endocrinology 143(10) (2002) 4104-12.10.1210/en.2002-220087. 
[13] V. Chandrashekar, D. Zaczek, A. Bartke, The consequences of altered somatotropic system on reproduction, Biol Reprod 71(1) (2004) 17-27.10.1095/biolreprod.103.027060.

[14] D. Zaczek, J. Hammond, L. Suen, S. Wandji, D. Service, A. Bartke, V. Chandrashekar, K. Coschigano, J. Kopchick, Impact of growth hormone resistance on female reproductive function: new insights from growth hormone receptor knockout mice, Biol Reprod 67(4) (2002) 111524.10.1095/biolreprod67.4.1115.

[15] A. Schneider, X. Zhi, F. Moreira, T. Lucia, Jr., R.G. Mondadori, M.M. Masternak, Primordial follicle activation in the ovary of Ames dwarf mice, J Ovarian Res 7 (2014) 120.10.1186/s13048-014-0120-4.

[16] T.D. Saccon, F. Moreira, L.A. Cruz, R.G. Mondadori, Y. Fang, C.C. Barros, L. Spinel, A. Bartke, M.M. Masternak, A. Schneider, Ovarian aging and the activation of the primordial follicle reserve in the long-lived Ames dwarf and the short-lived bGH transgenic mice, Mol Cell Endocrinol 455 (2017) 23-32.10.1016/j.mce.2016.10.015.

[17] T.D. Saccon, F. Moreira, L.A. Cruz, R.G. Mondadori, Y. Fang, C.C. Barros, L. Spinel, A. Bartke, M.M. Masternak, A. Schneider, Ovarian aging and the activation of the primordial follicle reserve in the long-lived Ames dwarf and the short-lived bGH transgenic mice, Molecular and cellular endocrinology (2016).10.1016/j.mce.2016.10.015.

[18] N. Saini, D.A. Gordenin, Somatic mutation load and spectra: A record of DNA damage and repair in healthy human cells, Environ Mol Mutagen 59(8) (2018) 672-686.10.1002/em.22215.

[19] T. Chiang, R.M. Schultz, M.A. Lampson, Meiotic origins of maternal age-related aneuploidy, Biol Reprod 86(1) (2012) 1-7.10.1095/biolreprod.111.094367.

[20] L.M. Mehlmann, Stops and starts in mammalian oocytes: recent advances in understanding the regulation of meiotic arrest and oocyte maturation, Reproduction 130(6) (2005) 7919.10.1530/rep.1.00793.

[21] S. Titus, F. Li, R. Stobezki, K. Akula, E. Unsal, K. Jeong, M. Dickler, M. Robson, F. Moy, S. Goswami, K. Oktay, Impairment of BRCA1-related DNA double-strand break repair leads to ovarian aging in mice and humans, Sci Transl Med 5(172) (2013) 172ra21.10.1126/scitranslmed.3004925.

[22] I. Adriaens, J. Smitz, P. Jacquet, The current knowledge on radiosensitivity of ovarian follicle development stages, Hum Reprod Update 15(3) (2009) 35977.10.1093/humupd/dmn063.

[23] M. Kirk, M.F. Lyon, Induction of congenital anomalies in offspring of female mice exposed to varying doses of X-rays, Mutation research 106(1) (1982) 73-83.

[24] C.J. Bakkenist, M.B. Kastan, DNA damage activates ATM through intermolecular autophosphorylation and dimer dissociation, Nature 421(6922) (2003) 499506.10.1038/nature01368. 
[25] S. Burma, B.P. Chen, M. Murphy, A. Kurimasa, D.J. Chen, ATM phosphorylates histone H2AX in response to DNA double-strand breaks, The Journal of biological chemistry 276(45) (2001) 42462-7.10.1074/jbc.C100466200.

[26] J.K. Collins, K.T. Jones, DNA damage responses in mammalian oocytes, Reproduction 152(1) (2016) R15-22.10.1530/rep-16-0069.

[27] A. Bektas, S.H. Schurman, R. Sen, L. Ferrucci, Aging, inflammation and the environment, Exp Gerontol 105 (2018) 10-18.10.1016/j.exger.2017.12.015.

[28] Y.F. Chen, S. Pandey, C.H. Day, A.Z. Jiang, T.J. Ho, R.J. Chen, V.V. Padma, W.W. Kuo, C.Y. Huang, Synergistic effect of HIF-1alpha and FoxO3a trigger cardiomyocyte apoptosis under hyperglycemic ischemia condition, J Cell Physiol 233(4) (2018) 3660$3671.10 .1002 /$ jcp.26235.

[29] A. Schneider, S.J. Matkovich, T. Saccon, B. Victoria, L. Spinel, M. Lavasani, A. Bartke, P. Golusinski, M.M. Masternak, Ovarian transcriptome associated with reproductive senescence in the long-living Ames dwarf mice, Mol Cell Endocrinol 439 (2017) 328336.10.1016/j.mce.2016.09.019.

[30] D.E. Berryman, E.O. List, K.T. Coschigano, K. Behar, J.K. Kim, J.J. Kopchick, Comparing adiposity profiles in three mouse models with altered GH signaling, Growth Horm IGF Res 14(4) (2004) 309-18.10.1016/j.ghir.2004.02.005.

[31] Z. Wang, K.A. Al-Regaiey, M.M. Masternak, A. Bartke, Adipocytokines and lipid levels in Ames dwarf and calorie-restricted mice, J Gerontol A Biol Sci Med Sci 61(4) (2006) 32331.10.1093/gerona/61.4.323.

[32] M.M. Masternak, A. Bartke, Growth hormone, inflammation and aging, Pathobiol Aging Age Relat Dis 2 (2012).10.3402/pba.v2i0.17293.

[33] E.C. Turner, J. Hughes, H. Wilson, M. Clay, K.J. Mylonas, T. Kipari, W.C. Duncan, H.M. Fraser, Conditional ablation of macrophages disrupts ovarian vasculature, Reproduction 141(6) (2011) 821-31.10.1530/rep-10-0327.

[34] Z. Durackova, Some current insights into oxidative stress, Physiol Res 59(4) (2010) 459-69.

[35] N. Khansari, Y. Shakiba, M. Mahmoudi, Chronic inflammation and oxidative stress as a major cause of age-related diseases and cancer, Recent Pat Inflamm Allergy Drug Discov 3(1) (2009) 73-80.

[36] A.N. Hirshfield, Development of follicles in the mammalian ovary, Int Rev Cytol 124 (1991) 43-101.10.1016/s0074-7696(08)61524-7.

[37] D. Adhikari, K. Liu, Molecular mechanisms underlying the activation of mammalian primordial follicles, Endocr Rev 30(5) (2009) 438-64.10.1210/er.2008-0048. 
[38] H. Zhang, S. Risal, N. Gorre, K. Busayavalasa, X. Li, Y. Shen, B. Bosbach, M. Brannstrom, K. Liu, Somatic cells initiate primordial follicle activation and govern the development of dormant oocytes in mice, Curr Biol 24(21) (2014) 2501-8.10.1016/j.cub.2014.09.023.

[39] Y. Munakata, M. Ueda, R. Kawahara-Miki, K. Kansaku, N. Itami, K. Shirasuna, T. Kuwayama, H. Iwata, Follicular factors determining granulosa cell number and developmental competence of porcine oocytes, J Assist Reprod Genet 35(10) (2018) 18091819.10.1007/s10815-018-1247-9.

[40] M. Laplante, D.M. Sabatini, mTOR signaling in growth control and disease, Cell 149(2) (2012) 274-93.10.1016/j.cell.2012.03.017.

[41] P. Reddy, L. Liu, D. Adhikari, K. Jagarlamudi, S. Rajareddy, Y. Shen, C. Du, W. Tang, T. Hamalainen, S.L. Peng, Z.J. Lan, A.J. Cooney, I. Huhtaniemi, K. Liu, Oocyte-specific deletion of Pten causes premature activation of the primordial follicle pool, Science 319(5863) (2008) 611-3.10.1126/science.1152257.

[42] G.B. John, T.D. Gallardo, L.J. Shirley, D.H. Castrillon, Foxo3 is a PI3K-dependent molecular switch controlling the initiation of oocyte growth, Dev Biol 321(1) (2008) 197204.10.1016/j.ydbio.2008.06.017.

[43] A. Louis, A. Bartke, M.M. Masternak, Effects of growth hormone and thyroxine replacement therapy on insulin signaling in Ames dwarf mice, J Gerontol A Biol Sci Med Sci 65(4) (2010) 344-52.10.1093/gerona/glq018.

[44] M.M. Masternak, J.A. Panici, F. Wang, Z. Wang, A. Spong, The effects of growth hormone (GH) treatment on GH and insulin/IGF-1 signaling in long-lived Ames dwarf mice, J Gerontol A Biol Sci Med Sci 65(1) (2010) 24-30.10.1093/gerona/glp172.

[45] A. Gesing, K.A. Al-Regaiey, A. Bartke, M.M. Masternak, Growth hormone abolishes beneficial effects of calorie restriction in long-lived Ames dwarf mice, Exp Gerontol 58 (2014) 219-29.10.1016/j.exger.2014.08.010.

[46] M.E. Skaznik-Wikiel, D.C. Swindle, A.A. Allshouse, A.J. Polotsky, J.L. McManaman, High-Fat Diet Causes Subfertility and Compromised Ovarian Function Independent of Obesity in Mice, Biol Reprod 94(5) (2016) 108.10.1095/biolreprod.115.137414.

[47] C.A. Schneider, W.S. Rasband, K.W. Eliceiri, NIH Image to ImageJ: 25 years of image analysis, Nat Methods 9(7) (2012) 671-5.

[48] H.M. Brown-Borg, K.E. Borg, C.J. Meliska, A. Bartke, Dwarf mice and the ageing process, Nature 384(6604) (1996) 33.10.1038/384033a0.

[49] S.P. Jackson, J. Bartek, The DNA-damage response in human biology and disease, Nature 461(7267) (2009) 1071-8.10.1038/nature08467.

[50] J. Bartek, J. Lukas, DNA damage checkpoints: from initiation to recovery or adaptation, Curr Opin Cell Biol 19(2) (2007) 238-45.10.1016/j.ceb.2007.02.009. 
[51] J. Smith, L.M. Tho, N. Xu, D.A. Gillespie, The ATM-Chk2 and ATR-Chk1 pathways in DNA damage signaling and cancer, Adv Cancer Res 108 (2010) 73-112.10.1016/b978-0-12380888-2.00003-0.

[52] H.C. Reinhardt, M.B. Yaffe, Kinases that control the cell cycle in response to DNA damage: Chk1, Chk2, and MK2, Curr Opin Cell Biol 21(2) (2009) 245-55.10.1016/j.ceb.2009.01.018.

[53] P.S. Burgoyne, S.K. Mahadevaiah, J.M. Turner, The management of DNA double-strand breaks in mitotic G2, and in mammalian meiosis viewed from a mitotic G2 perspective, Bioessays 29(10) (2007) 974-86.10.1002/bies.20639.

[54] A. Podlutsky, M.N. Valcarcel-Ares, K. Yancey, V. Podlutskaya, E. Nagykaldi, T. Gautam, R.A. Miller, W.E. Sonntag, A. Csiszar, Z. Ungvari, The GH/IGF-1 axis in a critical period early in life determines cellular DNA repair capacity by altering transcriptional regulation of DNA repair-related genes: implications for the developmental origins of cancer, Geroscience 39(2) (2017) 147-160.10.1007/s11357-017-9966-x.

[55] D. Zhang, X. Zhang, M. Zeng, J. Yuan, M. Liu, Y. Yin, X. Wu, D.L. Keefe, L. Liu, Increased DNA damage and repair deficiency in granulosa cells are associated with ovarian aging in rhesus monkey, Journal of assisted reproduction and genetics 32(7) (2015) 106978.10.1007/s10815-015-0483-5.

[56] Y. Fukumatsu, H. Katabuchi, M. Naito, M. Takeya, K. Takahashi, H. Okamura, Effect of macrophages on proliferation of granulosa cells in the ovary in rats, J Reprod Fertil 96(1) (1992) 241-9.10.1530/jrf.0.0960241.

[57] R.Z. Spaczynski, A. Arici, A.J. Duleba, Tumor necrosis factor-alpha stimulates proliferation of rat ovarian theca-interstitial cells, Biol Reprod 61(4) (1999) 9938.10.1095/biolreprod61.4.993.

[58] M. Nakayama, N. Manabe, N. Inoue, T. Matsui, H. Miyamoto, Changes in the expression of tumor necrosis factor (TNF) alpha, TNFalpha receptor (TNFR) 2, and TNFR-associated factor 2 in granulosa cells during atresia in pig ovaries, Biol Reprod 68(2) (2003) 5305.10.1095/biolreprod.102.004820.

[59] D. Stirling, M.R. Waterman, E.R. Simpson, Expression of mRNA encoding basic fibroblast growth factor (bFGF) in bovine corpora lutea and cultured luteal cells, J Reprod Fertil 91(1) (1991) 1-8.10.1530/jrf.0.0910001.

[60] D.L. McClelland Descalzo, T.S. Satoorian, L.M. Walker, N.R. Sparks, P.Y. Pulyanina, N.I. Zur Nieden, Glucose-Induced Oxidative Stress Reduces Proliferation in Embryonic Stem Cells via FOXO3A/beta-Catenin-Dependent Transcription of p21(cip1), Stem Cell Reports 7(1) (2016) 55-68.10.1016/j.stemcr.2016.06.006.

[61] K.T. Coschigano, A.N. Wetzel, N. Obichere, A. Sharma, S. Lee, R. Rasch, M.M. Guigneaux, A. Flyvbjerg, T.G. Wood, J.J. Kopchick, Identification of differentially expressed genes in the kidneys of growth hormone transgenic mice, Growth Horm IGF Res 20(5) (2010) 345-55.10.1016/j.ghir.2010.06.001. 
540 [62] J. Ding, D.E. Berryman, J.J. Kopchick, Plasma proteomic profiles of bovine growth 541 hormone transgenic mice as they age, Transgenic Res 20(6) (2011) 1305-20.10.1007/s11248-

542 011-9499-5.

543 [63] P.E. Cohen, L. Zhu, K. Nishimura, J.W. Pollard, Colony-stimulating factor 1 regulation of 544 neuroendocrine pathways that control gonadal function in mice, Endocrinology 143(4) (2002) $545 \quad$ 1413-22.10.1210/endo.143.4.8754.

546 [64] J.J. Eppig, F. Chesnel, Y. Hirao, M.J. O'Brien, F.L. Pendola, S. Watanabe, K. 547 Wigglesworth, Oocyte control of granulosa cell development: how and why, Hum Reprod 12(11 548 Suppl) (1997) 127-32.

549 [65] Y.Q. Su, K. Sugiura, J.J. Eppig, Mouse oocyte control of granulosa cell development and 550 function: paracrine regulation of cumulus cell metabolism, Semin Reprod Med 27(1) (2009) 32$551 \quad 42.10 .1055 / \mathrm{s}-0028-1108008$.

552 [66] Y. Munakata, T. Ichinose, K. Ogawa, N. Itami, H. Tasaki, K. Shirasuna, T. Kuwayama, H. 553 Iwata, Relationship between the number of cells surrounding oocytes and energy states of 554 oocytes, Theriogenology 86(7) (2016) 1789-1798.e1.10.1016/j.theriogenology.2016.05.036. 